\title{
Efficiency of encounter-controlled reaction between diffusing reactants in a finite lattice: topology and boundary effects.
}

\author{
Jonathan L. Bentz and John J. Kozak \\ Department of Chemistry, Iowa State University, Ames, Iowa 50011-3111 \\ E. Abad and G. Nicolis \\ Center for Nonlinear Phenomena and Complex Systems, \\ Université Libre de Bruxelles, C. P. 231, \\ Bd. Du Triomphe, 1050 Brussels, Belgium
}

(Dated: November 16, 2018)

\begin{abstract}
The role of dimensionality (Euclidean versus fractal), spatial extent, boundary effects and system topology on the efficiency of diffusion-reaction processes involving two simultaneously-diffusing reactants is analyzed. We present numerically-exact values for the mean time to reaction, as gauged by the mean walklength before reactive encounter, obtained via application of the theory of finite Markov processes, and via Monte Carlo simulation. As a general rule, we conclude that for sufficiently large systems, the efficiency of diffusion-reaction processes involving two synchronously diffusing reactants (two-walker case) relative to processes in which one reactant of a pair is anchored at some point in the reaction space (one walker plus trap case) is higher, and is enhanced the lower the dimensionality of the system. This differential efficiency becomes larger with increasing system size and, for periodic systems, its asymptotic value may depend on the parity of the lattice. Imposing confining boundaries on the system enhances the differential efficiency relative to the periodic case, while decreasing the absolute efficiencies of both two-walker and one walker plus trap processes. Analytic arguments are presented to provide a rationale for the results obtained. The insights afforded by the analysis to the design of heterogeneous catalyst systems is also discussed.
\end{abstract}

PACS numbers: 05.40.-a; 82.20.Fd

Keywords: Diffusion-controlled reactions; lattice walks; trapping problems 


\section{INTRODUCTION}

The influence of the interplay between spatial extent and system dimensionality on the reaction efficiency when reactants are undergoing random displacements on a finite lattice, with an irreversible reaction occurring on first encounter has attracted considerable interest over the last years 1, 2, 3, 4]. Whereas there is a vast literature dealing with the situation where one of the two reaction partners is anchored at some point in the reaction space [2, 3], a novel feature addressed more recently is when both reaction partners are allowed to diffuse simultaneously [1, 4]. This corresponds to the kinetic scheme

$$
A+A \rightarrow 2 S
$$

where $A$ denotes a site occupied by a diffusing reactant molecule and $S$ a free site, as opposed to the scheme

$$
A+T \rightarrow S+T
$$

in which $T$ denotes a site occupied by an immobile target molecule (trap). In this paper, we shall refer to the scheme described by Eq. (11) as the "two-walker (2W) case", while the kinetics described by Eq. (2) will be termed "one-walker plus trap (1WT) case".

In Refs. [1, 4], particular attention was focused on processes taking place on square-planar lattices subject to various boundary conditions. It was found that significant differences in reaction efficiency resulted depending on whether one or both reactants were diffusing. The objective of the present study is to inquire to what extent these results are generic and, if so, how they depend on the geometry of the support. The latter will be characterized, in turn, by the size, the embedding dimension, the intrinsic dimensionality, and by the topological invariants. Among these, for two-dimensional objects (surfaces) the Euler characteristic $\chi=F-E+V$ where $F$ is the number of faces, $E$ the number of edges and $V$ the number of vertices is especially significant. In each case, the role of the boundary conditions will also be assessed.

The mean walklength of a diffusing species before encountering a coreactant is a natural measure of the time scale of a diffusion-reaction process. Let $\langle n\rangle_{1}$ be the mean walklength before the irreversible reaction takes place in the $1 \mathrm{WT}$ case (Eq. (2)). Let $\langle n\rangle_{2}$ be the corresponding quantity for the $2 \mathrm{~W}$ case (Eq. (1)). The quantities $\langle n\rangle_{1,2}$ are obtained by averaging over statistical realizations comprising all different initial configurations of both reaction partners. The smaller the value of $\langle n\rangle_{1,2}$, the higher the efficiency of the reaction.

A point that will recur frequently in the following is that the reaction in the $2 \mathrm{~W}$ case can occur via two different channels. In the first scenario, the two reaction partners happen to occupy adjacent sites on the lattice, and (with a certain probability) in their next, mutual displacement they undergo a collision. In the second channel, an intervening lattice site separates the two reactants and, in their subsequent motion (again, with a certain probability), both attempt to occupy that same (vacant) lattice site. As we shall see, one or both of these reaction channels can pertain, depending on the choice of boundary conditions and the parity of the total number of lattice sites $N$. Since both $\langle n\rangle_{1}$ and $\langle n\rangle_{2}$ depend on the characteristics of the lattice and on the boundary conditions, it will therefore be more appropriate to choose as measure of the relative efficiency of the $2 \mathrm{~W}$ process the ratio $\Gamma \equiv\langle n\rangle_{1} /\langle n\rangle_{2}$.

There are both theoretical and experimental reasons for studying the influence of geometry and boundary conditions on the efficiency of diffusion-reaction processes. First, one would like to isolate effects that are independent of the boundaries of the domain in which 
the diffusion-reaction processes take place, and for this, periodic boundary conditions are usually imposed. Over the last several years, however, there has been an avalanche of experimental work published on the study of diffusion-reaction processes in microheterogeneous media [5] (micelles, clays, zeolites, etc.), with systems of finite extent (nanosystems) showing non-classical behavior (i.e., departures from "mean field" behavior). Therefore, in the present study we have also examined in tandem with periodic boundary conditions, systems subject to confining boundary conditions. Specifically, in adopting confining boundary conditions, one imposes the condition that if a diffusing particle attempts to exit the lattice from a given boundary site, it is simply reset at that boundary site.

The calculations performed in this paper are of two kinds. First, we use the theory of finite Markov processes to compute $\langle n\rangle_{1}$ and $\langle n\rangle_{2}$, for small, finite lattices subject either to periodic or confining boundary conditions. The advantage of this approach is that one obtains numerically-exact solutions to the problem under study and, in certain cases, one can construct closed-form analytic solutions. Mirroring this, we also perform Monte Carlo calculations, first validated by comparison with the Markov results and then used to analyze systems of large spatial extent.

The plan of this paper is as follows. In Secs. 24, we consider successively lattices of Euclidean dimension $d=1, d=2$ and $d=3$, as well as the Sierpinski gasket, a lattice of fractal dimension $D=\ln 3 / \ln 2$. The influence of size effects, boundary conditions and other related topological features such as the connectivity and the Euler characteristic is discussed in each dimension. The main conclusions are summarized in Sec. 5 .

\section{EUCLIDEAN DIMENSION, $d=1$}

The 1WT problem on a $d=1$ finite lattice subject to periodic boundary conditions was solved by Montroll [6] analytically. The result he found was:

$$
\langle n\rangle_{1}=\frac{N(N+1)}{6} .
$$

where $N$ is the total number of sites in the lattice.

The solution for the case of two walkers in $d=1$ for a lattice subject to periodic boundary conditions can be obtained in closed form by formulating the problem as a classical ruin problem and solving the correspondence difference equations [7]. The result is

$$
\langle n\rangle_{2}=\left\{\begin{array}{ll}
N(N+1)(N+2) / 12(N-1) & \text { for } N \text { even } \\
(N+1)(N+3) / 12 & \text { for } N \text { odd }
\end{array} .\right.
$$

This result can also be obtained via the theory of finite Markov processes by calculating numerically-exact values of $\langle n\rangle_{2}$ for a series of $d=1$ lattices. From these results patterns can be recognized from which one can construct the above closed-form analytic solution.

We notice a difference in the expression for $\langle n\rangle_{2}$ for even and odd values of $N$ also found in Refs. [1, 4] and which will turn out to become more pronounced in higher dimensions. We find that this qualitative difference in behavior between even and odd lattices is always manifested if periodic boundary conditions are considered, but not if confining boundary conditions are imposed. This behavior can be rationalized by examination of small odd and even lattices. The key to this difference is in the reaction mechanism, which happens either via nearest-neighbor collision ( $\mathrm{NNC}$ ) or via same-site occupation (SSO). 
Consider the two periodic lattices of size $N=3$ and $N=4$ shown in Figs. 17a and 1p. For $N=3$, assume that we place a walker on site 1 and another one on site 3 . Concerned only with the concerted motions leading to reaction, one can see that reaction takes place by SSO if both walkers jump to site 2 , or by NNC if they attempt to exchange positions. Thus, both reaction channels are open. Now consider the periodic $N=4$ lattice and place again the walkers on sites 1 and 3. The reaction can proceed by SSO if both walkers jump to site 2 or site 4 after one time step. Otherwise, they will always be two sites apart, essentially following each other in the lattice. This means that starting with the initial configuration of walkers on sites 1 and 3, the only allowed reaction channel is SSO. On the other hand, if sites 1 and 2 are chosen as the initial positions of the walkers, or more generally any adjacent pair of sites, reaction can only occur by NNC. This is true because any concerted motion will either lead to NNC or the walkers will remain nearest-neighbors after each time step. More generally, it is easily seen that for an even lattice only one of the reaction channels is active for any given initial configuration, whereas for odd lattices, both can take place. This phenomenon will hereafter be referred to as the "even-odd effect".

We next consider the $1 \mathrm{WT}$ and the $2 \mathrm{~W}$ problems in a $d=1$ lattice subject to confining boundary conditions. From Markov theory, one obtains for the 1WT:

$$
\langle n\rangle_{1}=\frac{N(N+1)}{3} .
$$

According to this formula, the reaction efficiency is reduced by a factor of 2 with respect to the periodic case described by Eq. (3) for all system sizes. The reason is that the lattice with confining boundaries and a trap $T$ can be decomposed into two disconnected periodic lattices, each of them with a trap, since the parts of the lattice on either side of $T$ do not communicate $^{1}$. This equivalence is illustrated in Fig. 2 a for a confining lattice with $N=4$. $^{2}$ The weighted size of all equivalent periodic lattices resulting from the different positions of the trapping site $T$ is larger than $N$. Similar arguments are expected to apply in higher dimensions. Thus, confinement always reduces the efficiency of the reaction.

As for the $2 \mathrm{~W}$ problem, no closed form expression similar to Eq. (41) has yet been derived for the case of confining boundary conditions. However, values of $\langle n\rangle_{2}$ for this case could be calculated numerically using both the Markov method and Monte Carlo simulations. As in the $1 \mathrm{WT}$ case, the efficiency is smaller than in the periodic lattice case, but the increase in $\langle n\rangle_{2}$ when confinement is imposed is smaller than the factor of 2 found in the $1 \mathrm{~W}$ case for all values of the lattice size $N$ larger than $t_{w o}^{2}$, approaching a value close to 1.70 from below in the asymptotic limit (not shown). Once more, an equivalence with the periodic case can be established here. However, the difference with the 1WT case is that now the size of the equivalent periodic lattices fluctuates in time, as it depends on the instantaneous distance. These lattice size fluctuations possibly explain why the loss of efficiency is smaller in the $2 \mathrm{~W}$ case when one switches from periodic to confining boundary conditions.

We notice that no even-odd effect is to be expected in the confining case. This is illustrated in Fig. 2b with two walkers initially placed on sites 1 and 3. Obviously SSO occurs if both walkers jump to site 2 . If both walkers jump to the left, then the walker on site 3 will jump to site 2 , but the walker on site 1 will not move. Both walkers occupy now adjacent

\footnotetext{
${ }^{1}$ For the special case where the trap is placed at an edge site, the confining lattice reduces to a single rather than to two periodic lattices.

${ }^{2}$ For $N=2$, a confining lattice is even more effective than a periodic one.
} 
sites and NNC becomes possible at the next time step. For the other symmetry-distinct initial configuration with walkers at sites 1 and 2, one can formulate an analogous sequence of jumps leading to SSO. Thus, for a given initial condition, the imposition of confining boundary conditions allows both reaction channels on an even-site lattice, whereas with periodic boundary conditions only one of them is realized.

Figure 3 depicts the behavior of the relative efficiency $\Gamma$ defined in the Introduction as a function of the lattice size $N$. It reveals an important feature, seen for all Euclidean lattices studied regardless of dimension or class of boundary conditions ${ }^{3}$. The curve $\Gamma$ vs. $N$ exhibits a steep increase initially with increase in $N$ but then it "flattens out" and appears to be saturating at a particular limiting value $\Gamma_{\infty} \equiv \lim _{N \rightarrow \infty} \Gamma$. The limiting value $\Gamma_{\infty}$ is different for different dimensions, but the same steep increase followed by a very gradual increase to a limiting value is found. For the periodic case in $d=1$, the numerical evidence suggests that the curve is approaching a value close to 2 ; upon inspection of the analytic form of the solutions given by Eqs. (13) and (41) one sees that the value in the limit of large $N$ is exactly 2. This is related to the fact that, due to the translational invariance of the lattice, the $2 \mathrm{~W}$ problem can be reduced to an equivalent $1 \mathrm{WT}$ problem, and the effective diffusion coefficient for the latter becomes twice as large in the large $N$ limit [7].

A second feature revealed by a closer examination of the analytic form for $\Gamma$ in the periodic case is that the increase with size is staircase-like since $\Gamma$ takes the same value for two consecutive odd and even values of $N$ 7]. This consequence of the even-odd effect characteristic of the periodic case is not seen at the scale of resolution of Fig. 3 and is actually unimportant for the qualitative behavior over sufficiently large $N$ intervals. Unlike toroidal (periodic) lattices in higher dimensions, the even-odd effect in one dimension does not lead to different asymptotic values $\Gamma_{\infty}^{e v e n}$ and $\Gamma_{\infty}^{o d d}$ depending on whether only even or only odd lattices are considered for the computation of the respective walklengths.

In general, plotting the $\langle n\rangle_{1}$ vs. the $\langle n\rangle_{2}$ data for sufficiently large values of $N$, as displayed in Fig. 4, is a convenient way of determining the saturation value $\Gamma_{\infty}$. The slope value from a least-squares curve fit for periodic lattices is 1.97, in good agreement with the exact analytical value, and 2.35 for confining lattices.

\section{EUCLIDEAN DIMENSION, $d=2$}

A square-planar lattice with periodic boundary conditions is topologically equivalent to a torus and has an Euler characteristic $\chi$ of 0 . Results in $d=2$ have already been reported for the case of a square-planar lattice subject to periodic boundary conditions [1]. Figure 5 shows clearly the behavior of $\Gamma$ in the large lattice limit, which is different for odd and even lattices. In Ref. [1], analytical evidence was provided to show that the asymptotic limit of $\Gamma$ for odd lattices is $\Gamma_{\infty}^{o d d}=\sqrt{2}$.

As mentioned in the Introduction, surfaces are characterized topologically not only by their Euclidean dimension but also by their Euler characteristic. Thus, it is also of interest to consider non-toroidal surfaces with Euler characteristic $\chi \neq 0$. In particular, for the sphere and the family of polyhedra homeomorphic to it, one has $\chi=2$. We shall consider two examples, the first of which is the case of diffusion-reaction processes on the surface of the Platonic solids. One of the reasons motivating the study of such polyhedral systems

\footnotetext{
${ }^{3}$ The sole exception is the $d=3$ cubic lattice subject to confining boundary conditions; see Section 4.1
} 
is that they mimic some features of real-world solid catalysts where the reaction takes place on particular crystallographic faces of the solid. In earlier work [8], the vertices of the Platonic solids were used as the allowable particle positions. The movement of each particle was permitted along any adjoining edge. In the present study, we report the results of the calculation using the faces of the Platonic solids; the particle moves from face to adjacent face, crossing only one edge at each time step. The relationship between these two calculations is reciprocal. In formal language, two polyhedra are dual if the number of faces of one of them equals the number of vertices of the other, and vice versa 9]. Recall that the tetrahedron has 4 faces and 4 vertices and both faces and vertices have a valency of 3 . The octahedron has 8 faces and 6 vertices, and the hexahedron (cube) has 6 faces and 8 vertices. The valency of the octahedron faces is 3 as is the valency of the cube vertices. Likewise, the valency of the octahedron vertices is 4 as is the valency of the cube faces. Performing the calculation on the faces of the octahedron is equivalent to the calculation on the vertices of the cube, and the same reciprocal relation holds true when examining the icosahedron and the dodecahedron. Table 1 shows results for $\langle n\rangle_{1}$ and $\langle n\rangle_{2}$ on the Platonic solids. As can be seen, with the exception of the octahedron, $\Gamma$ increases with $N$, in line with the results for the square planar lattice.

It is instructive to view the results of the Platonic solids in tandem with their planar analogues. The tetrahedron, octahedron and hexahedron can be placed in correspondence with periodic planar lattices with the same valency and same value of $N$; the dodecahedron and icosahedron have no periodic, planar analogues. In correspondence with the tetrahedron and octahedron we constructed hexagonal lattices for comparison, while the hexahedron was compared with a $2 \times 3$ square-planar lattice. The tetrahedron and hexahedron have smaller values than their planar analogues for the walklengths $\langle n\rangle_{1}$ and $\langle n\rangle_{2}$ but larger values of $\Gamma$. Surprisingly all the walklength values for the octahedron and the 8-site hexagonal lattice are identical. Upon further examination of this degeneracy, one finds that the values are identical because the connectivity of the octahedron is identical, site by site, with the connectivity of the 8-site periodic hexagonal lattice. These calculations show, surprisingly, that the connectivity of the lattice seems to play a relatively more important role than the Euler characteristic in determining the value of the mean walklength.

We also examined the influence of imposing confining boundary conditions on the planar lattice analogues of the Platonic solids. Owing to the fact that the connectivity of sites on the boundary of these (finite) lattices changes, the values of $\langle n\rangle_{1}$ and $\langle n\rangle_{2}$ change. The values of $\langle n\rangle_{2}$ for all three planar analogues are only slightly larger than for the case where periodic boundary conditions are imposed. On the other hand, the values of $\langle n\rangle_{1}$ are considerably larger than the values of $\langle n\rangle_{1}$ calculated for periodic boundary conditions; for the $N=4$ hexagonal lattice, the $N=8$ hexagonal lattice and the $N=2 \times 3$ square-planar lattice, the value of $\langle n\rangle_{1}$ increases by a factor of 3,4 and 2 , respectively. Thus, in small systems, changes in the boundary conditions have a significant effect on the calculation of $\langle n\rangle_{1}$, but less so on the calculation of $\langle n\rangle_{2}$. Overall, the influence of boundary conditions on both the $1 \mathrm{WT}$ and $2 \mathrm{~W}$ cases for these planar analogues of the Platonic solids is more drastic than is the case for a (small) $d=1$ lattice. This is reflected in the value of $\Gamma$, which increases by a larger factor here than in the $d=1$ case when confining boundary conditions are imposed.

In order to emulate large-size systems, we have studied diffusion-reaction processes on the surface of a cube, which is also of Euclidean dimension $d=2$ and Euler characteristic $\chi=2$. The particle is confined to the surface, and each face of the cube is divided into a $N \times N$ square planar grid so that there are $N \times N$ accessible sites on each face. The total 
number of sites is $N \times N \times 6$. Note that for $N=1$ the previous case of an hexahedron is recovered. The valency of each site is exactly four, keeping in mind that some movements of the walker will take it to a different face.

Figure 5 summarizes the $d=2$ results in the large lattice limit. It is seen that, on the surface of a cube, the differential efficiency $\Gamma$, given by the slope of the curve $\langle n\rangle_{1}$ vs. $\langle n\rangle_{2}$, is larger than on a periodic but smaller than on a confining square planar lattice. As expected, the efficiency of 1WT processes is once again decreased in a more pronounced way when confinement is imposed. The even-odd effect is not seen for processes on the surface of a cube or for planar lattices subject to confining boundary conditions. On the cube, an argument analogous to the one put forth for $d=1$ applies, except that the walkers on the cube surface have two additional degrees of freedom. However, this has no influence on the fact that, irrespective of the starting configuration, both NNC and SSO may still take place because of the the walkers' ability to traverse edges and thereby migrate to different faces of the cube.

\section{HIGHER DIMENSIONS AND FRACTAL STRUCTURES}

\subsection{Euclidean Dimension, $d=3$}

Numerically-exact results have been reported previously [10, 11] for the 1WT case on a $d=3$ cubic lattice subject to periodic boundary conditions. We have extended this study to calculate $\langle n\rangle_{1}$ and $\langle n\rangle_{2}$ for both periodic and confining boundary conditions by means of Monte Carlo simulations. In $d=3$, the even-odd effect is even more pronounced than in the square-planar lattice case, as may be seen in Fig. 6. From the least-square fits we infer $\Gamma_{\infty}^{e v e n}=0.72$ and $\Gamma_{\infty}^{o d d}=1.22$. This suggests that the even-odd effect is enhanced with increasing dimensionality of the hypercubic lattice. Note also that $\Gamma_{\infty}<1$ for even lattices, the only case where the differential efficiency goes below unity except for the octahedron.

For confining boundary conditions, another significant result for $d=3$ is shown in Fig. 7. As is evident, $\Gamma$ approaches its limiting value from above, and $\Gamma_{\infty}$ is found to be about 1.42 in this case. In all previous cases, namely the $d=1$ and $d=2$ cases with both types of boundary conditions, the graphs had the general features of Fig. 3. approaching $\Gamma_{\infty}$ from below.

\subsection{Fractal Dimension, $D=\ln 3 / \ln 2$}

From the results presented for the cases $d=1$, the $d=2$ square-planar lattice, and $d=3$ cubic lattice subject to confining boundary conditions, one can infer that, with increasing dimensionality d of the lattice, $\Gamma_{\infty}$ decreases monotonically taking the values $2.35,1.66$ and 1.42 , respectively, for $d=1,2$ and 3 . In order to assess further the role of dimensionality and of confinement, one can next consider the case of a Sierpinski gasket, whose ramified selfsimilar structure is described by an intrinsic non-integer dimension $D=\ln 3 / \ln 2 \simeq 1.585$. The gasket can be constructed hierarchically as a limit of successive generation gaskets. Each generation gasket is characterized by an index $i$. The $i=0$ generation is an equilateral triangle whose 3 vertices play the role of lattice sites, i.e. allowable reactant positions. The

$i=1$ generation can be constructed by appending to each of the bottom vertices of the primary triangle an additional equilateral triangle so that the upper vertices of the appended 
triangles are identical with the bottom vertices of the primary one and the appended triangles have a common bottom vertex. The $i=2$ generation is constructed by performing the same procedure on the resulting structure, i.e. appending twice the same structure at its bottom, and so on. Thus, at a given generation step, the number of identical substructures increases by three while the linear size doubles, yielding the above value of $D$ for the Sierpinski gasket obtained when $i \rightarrow \infty$. For each generation, the lattice sites are identified with the three apex vertices located at the outmost corners of the corresponding gasket and with the common vertices of any pair of adjacent triangles in the gasket. The number of sites in the $i$ th generation gasket is $N=N(i)=(3 / 2)\left(3^{i}+1\right)$.

The gasket shares some common features with a square-planar lattice. First, the embedding dimension is the same, viz. $d=2$. Secondly, except for the vertex sites on the gasket, the valency of all other sites is $v=4$; increasing the size of the gasket decreases the fraction of lattice sites not having a valency of 4 . All sites on a square-planar lattice subject to periodic boundary conditions have a valency $v=4$. On imposing confining boundary conditions on the square-planar lattice, all interior sites will have valency $v=4$, vertex sites will have valency $v=2$ and boundary sites will have valency $v=3$; the percentage of vertex and boundary sites on a square-planar lattices also decreases with increase in lattice size. Thus, both for the gasket and for a finite square-planar lattice subject to confining boundary conditions, the relative importance of interior sites on the statistics increases with increasing lattice size. Finally, one can easily convince oneself that in the $2 \mathrm{~W}$ case both the SSO and NNC channels can take place for any given configuration of the walkers on the gasket, so no even-odd effect is expected here either.

In view of the above resemblances, it is interesting to inquire to what extent the results obtained for the relative efficiency $\Gamma$ on the gasket are similar to those for the square lattice with confining boundary conditions. To this end, $\langle n\rangle_{1}$ and $\langle n\rangle_{2}$ and their ratio were calculated using the theory of finite Markov processes for $N=6,15$, and 42 and via Monte Carlo simulations for all generations up to $N=3282$. For the $1 \mathrm{WT}$ case, a closed-form analytic expression for the walklength is already available if the trap is maintained at one of the apex vertices of the $n$th generation gasket, namely [12]

$$
\langle n\rangle_{1, a}=\frac{3^{i} 5^{i+1}+4\left(5^{i}\right)-3^{i}}{3^{i+1}+1} .
$$

In performing Monte Carlo simulations, the amount of computing time can increase dramatically within increase in gasket size $N$ thus limiting the number of statistical realizations $n_{\text {real }}$. In the $1 \mathrm{WT}$ case, each realization comprises all sets of possible configurations for the walker and the trap, while in the $2 \mathrm{~W}$ case, each realization comprises all sets of possible configurations for the two indistinguishable walkers. The analytic expression of $\langle n\rangle_{1, a}$ given in Eq. (6) was used to test the accuracy of MC results for gasket sizes $N>123$, where the Markovian approach becomes cumbersome due to the size of the relevant transition matrices. It was found that, as $N$ is increased, the number of realizations required to reach a given accuracy in $\langle n\rangle_{1, a}$ with respect to the exact result given by (6) decreases significantly. For all values of $n_{\text {real }}$ given in Table 22 the relative error turned out to be $<0.4 \%$ (data not shown). Therefore, the results for $\langle n\rangle_{1}$ and $\langle n\rangle_{2}$ for large $N$ displayed in Table 2 seem reliable enough to describe correctly the qualitative behavior of the efficiencies.

Although the convergence to a hypothetical saturation value is very slow in $N$, one notices from Table 2 that $\Gamma$ appears to exceed the value 2 with increasing $N$, in contrast to the result $\Gamma_{\infty}=1.66$ for a confining square lattice. Assuming that $\Gamma$ keeps on increasing monotonically 
with the gasket size $N$, a question of interest is whether $\Gamma$ approaches a saturation value less than the value 2.35 found for a $d=1$ confining lattice, or whether the asymptotic value remains between the values 1.66 and 2.35, as one might expect in view of the monotonic decrease of $\Gamma_{\infty}$ with decreasing dimensionality referred earlier.

\section{CONCLUSIONS}

In this work, results, both analytic and numerical, on the role of boundary effects and of geometric factors such as size, dimensionality and topological invariants on the efficiency of encounter-controlled reactions have been obtained. Values for the mean walklengths $\langle n\rangle_{1}$ and $\langle n\rangle_{2}$ for the $1 \mathrm{WT}$ and the $2 \mathrm{~W}$ case as well as the relative efficiency $\Gamma$ have been computed both for large lattices and small size systems.

The relevance of considering in detail systems of restricted spatial extent is increasing, as it is nowadays realized that heterogenous catalytic processes of great importance take place on single crystallographic faces of solid catalysts where they can involve only a few tens of particles. Despite the fact that the numerical values obtained in this work for small systems are not universal, some universal trends of a different kind have nevertheless been observed, e.g. the dependence (increasing, monotonic, etc.) of $\Gamma$ on valency, connectivity and boundary conditions. In general, one has $\Gamma>1$, implying that the reactive efficiency of two moving reactants is greater than a diffusing plus an immobile one.

Turning now to large-size systems, our results show that $\Gamma$ increases with increasing lattice size until it reaches a well-defined limiting value $\Gamma_{\infty}$. For a given lattice geometry, this value decreases with increasing dimensionality. With the exception of the even cubic lattice, $\Gamma_{\infty}>1$.

For Euclidean lattices of square-planar or cubic symmetry and subject to periodic boundary conditions the value of $\Gamma_{\infty}^{o d d}$ is exactly 2 and $\sqrt{2}$ in one ${ }^{4}$ and two dimensions respectively, and about 1.22 in three dimensions. One is tempted to advance that this last number is actually within the precision afforded by the simulations just $\sqrt{(3 / 2)}$. Now, $1, \sqrt{2}$ and $\sqrt{3}$ are the natural metrics of the lattices here considered in, respectively, 1, 2 and 3 dimensions. The results for $\Gamma_{\infty}^{o d d}$ could then be particular cases of a universal expression: the ratio of the maximum distance that two simultaneously moving reactants can traverse in one time unit before they react or find themselves in nearest neighbor positions, over the analogous quantity for the one reactant plus trap case. Further work is necessary to assert the validity of this conjecture and to understand why it manifests itself only for odd lattices.

For a given system size, the $1 \mathrm{WT}$ and the $2 \mathrm{~W}$ reaction efficiency becomes less efficient when confinement is introduced, but the decrease in efficiency is smaller for the $2 \mathrm{~W}$ case, leading to an increased value of $\Gamma$ with respect to the periodic case. This boundary effect appears to become less significant with increasing system size, although it is not completely absent in the thermodynamic limit.

The questions raised in this work and the results obtained constitute potentially useful elements in the important problem of optimal design of the microreactors nowadays involved in chemical kinetics under nanoscale conditions. For instance, as seen in Sec. III, a small

\footnotetext{
${ }^{4}$ In the diffusive limit, it can be shown that the diffusion coefficient for the relative motion of both reactants is twice as large in the $2 \mathrm{~W}$ case [7]. Discrepancies from this value for small systems are due to the discreteness of the lattice. Possibly similar arguments hold in higher dimensions.
} 
catalytic surface in the form of a sphere or of a hexahedral surface homeomorphic to it $(\chi=2)$ would enhance the reaction efficiency as compared to a surface homeomorphic to a torus $(\chi=0)$. These observations highlight the need to incorporate in the design such aspects as the geometry of the microreactor, which can enhance an increasingly effective mixing of the reactants and hence an increased efficiency of the reaction itself. Finally, the role of the kinetics (linear vs. nonlinear) in modulating or enhancing the importance of such factors is certainly a problem worth addressing and this study is underway.

\section{Acknowledgments}

It is a pleasure to thank Dr. M. Plapp for valuable suggestions concerning the hierarchical construction of the Sierpinski gasket. This work is supported by a NATO Cooperative linkage grant PST.CLG.977780 and by the European Space Agency under contract number 90043.

[1] J. J. Kozak, C. Nicolis, G. Nicolis, J. Chem. Phys. 113 (2000) 8168.

[2] J. J. Kozak, Adv. Chem. Phys. 115 (2000) 245.

[3] G. H. Weiss, Aspects and Applications of the Random Walk, North-Holland, New York/Amsterdam, 1994.

[4] C. Nicolis, J. J. Kozak, G. Nicolis, J. Chem. Phys. 115 (2001) 663.

[5] A. V. Barzykin, K. Seki, M. Tachiya, Adv. Coll. Inter. Sci. 89-90 (2001) 47.

[6] E. Montroll, J. Math. Phys. 10 (1969) 753.

[7] E. Abad, G. Nicolis, J. L. Bentz, J. J. Kozak, Physica A (submitted).

[8] P. A. Politowicz, J. J. Kozak, Proc. Natl. Acad. Sci. USA. 84 (1987) 8175.

[9] Michael Henle, A Combinatorial Introduction to Topology, W.H. Freeman and Company, San Francisco, 1979.

[10] C. A. Walsh, J. J. Kozak, Phys. Rev. B. 26 (1982) 4166.

[11] C. A. Walsh, J. J. Kozak, Phys. Rev. Lett. 47 (1981) 1500.

[12] J. J. Kozak, V. Balakrishnan, Phys. Rev. E. 65 (2002) 021105. 


\section{Figure Captions}

Figure 1: Periodic $d=1$ lattices with a) $N=3$ and b) $N=4$.

Figure 2: a) Correspondence between confining and periodic boundary conditions for the two symmetry-distinct trap configurations in a confining 4-site lattice. Sites with same numbers are symmetry-equivalent. b) Four site confining lattice.

Figure 3: $\Gamma$ vs. $N$ in $d=1$. The curve for the periodic case is generated from the exact analytical results and the data for the confining case is from Monte Carlo simulations.

Figure 4: $\langle n\rangle_{1}$ vs. $\langle n\rangle_{2}$ in $d=1$. The slopes of the best-fit lines are: Periodic $=1.97$ and Confining $=2.35$. $N_{\min }=49$ for the periodic case $\left(N_{\min }\right.$ is the smallest data point used in the calculation of the best fit curve.) Both curves have $\mathrm{R}$ values of greater than 0.999 .

Figure 5: $\langle n\rangle_{1}$ vs. $\langle n\rangle_{2}$ in $d=2$. The slopes of the best-fit lines are: Confining $=1.66$, Even $=1.25$, Odd $=1.42$, Cube Surface $=1.47$. All data are calculated from simulations. The values of $N_{\min }$ are: Confining $=25$, Even $=100$, Odd $=81$, Cube Surface $=24$. All R values are greater than 0.999 .

Figure 6: $\langle n\rangle_{1}$ vs. $\langle n\rangle_{2}$ in $d=3$. The slopes of the best-fit lines are: Odd $=1.22$, Even $=$ 0.72 , Confining $=1.42$. All data are calculated from simulations. $N_{\min }=8$ for all the curves with all $\mathrm{R}$ values greater than 0.999 .

Figure 7: $\Gamma$ vs. $N$ in $d=3$ subject to confining boundary conditions; all data are calculated from simulations. 
a)

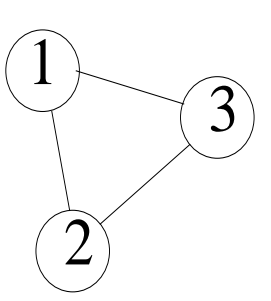

b)

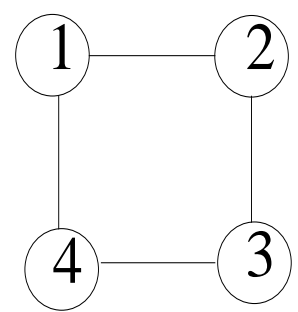

FIG. 1:
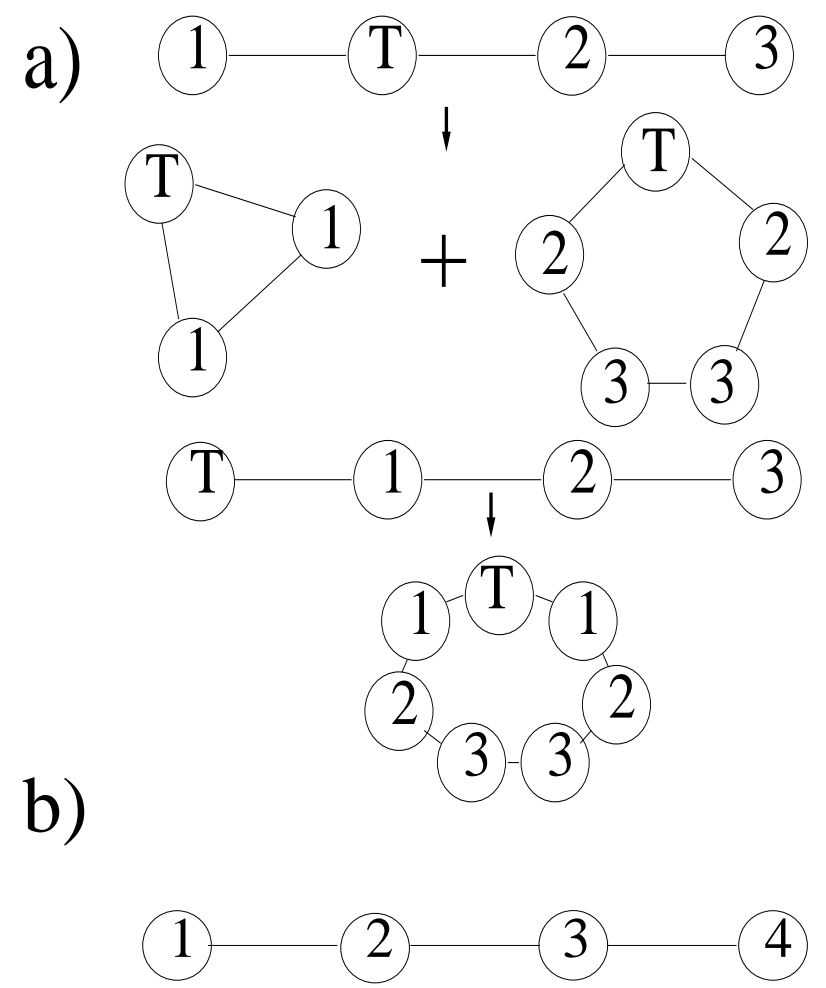

FIG. 2: 


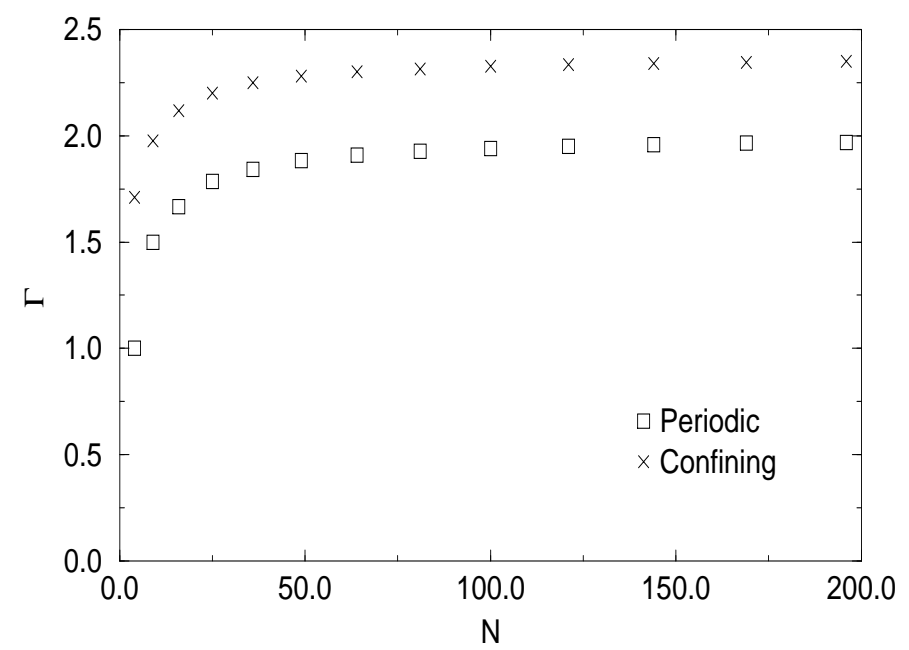

FIG. 3:

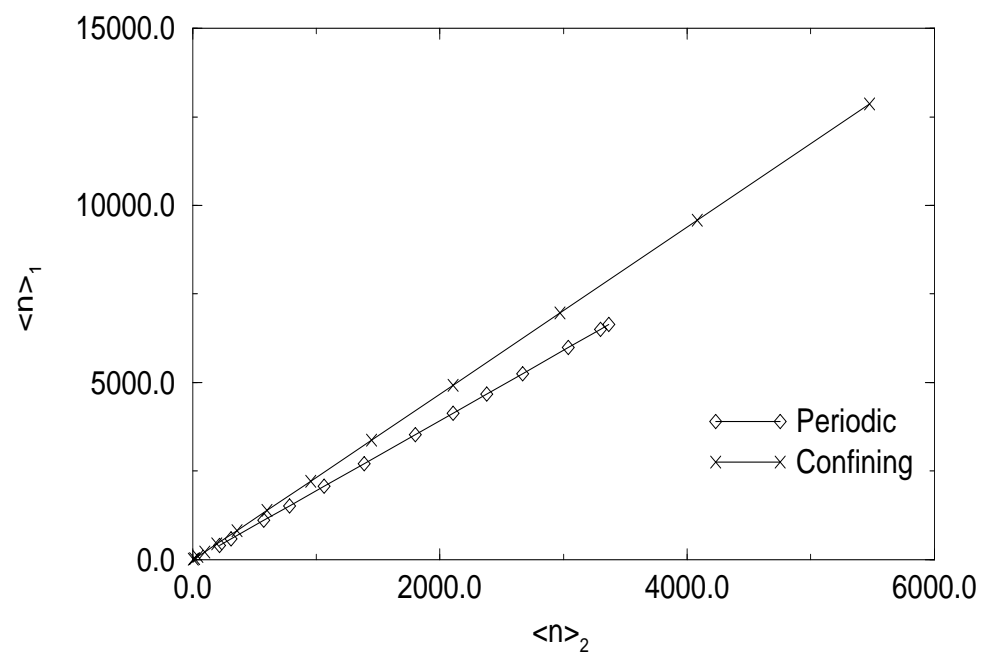

FIG. 4: 


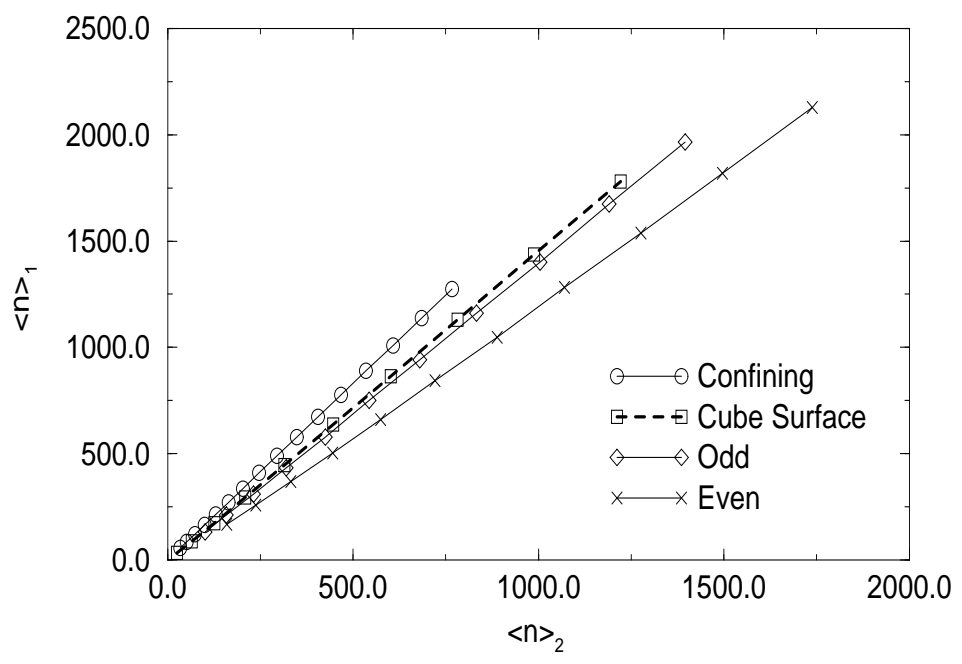

FIG. 5:

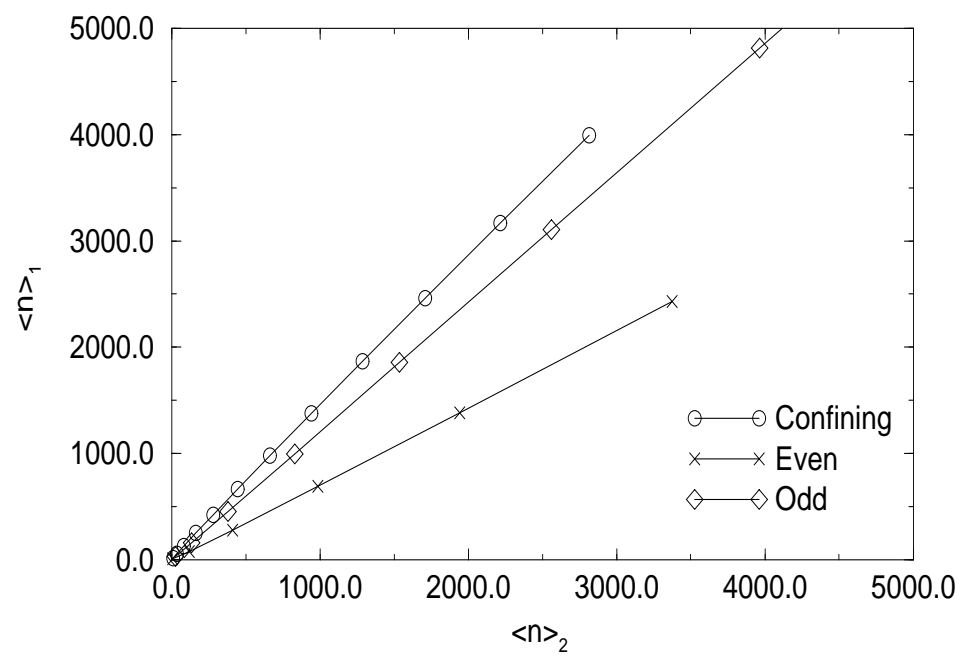

FIG. 6: 


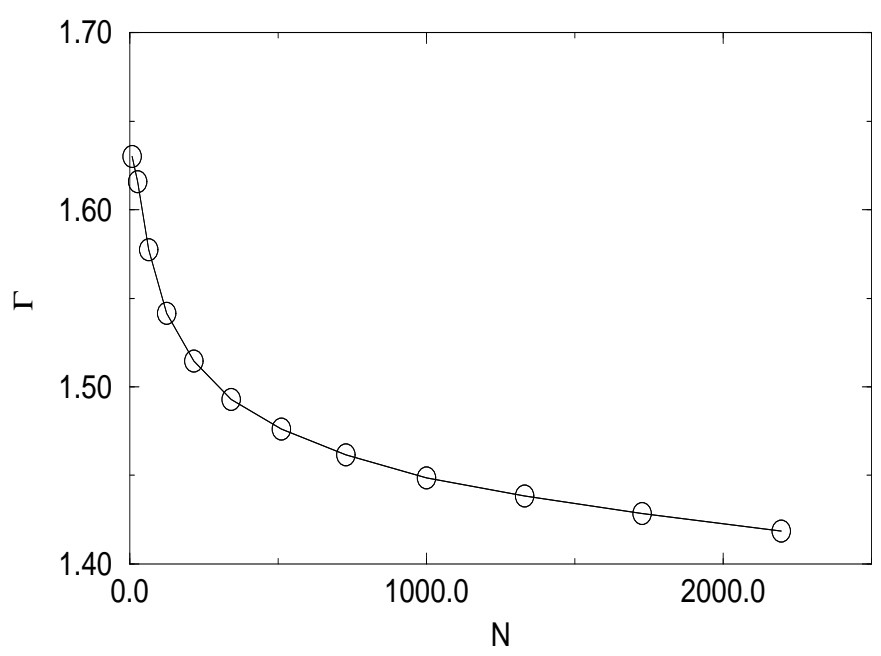

FIG. 7:

\begin{tabular}{c|ccccc}
\hline \hline Object & $N$ valency & $\langle n\rangle_{1}$ & $\langle n\rangle_{2}$ & $\Gamma$ \\
\hline Tetrahedron & 4 & 3 & 3 & 3 & 1 \\
Hexagonal Lattice & 4 & 3 & 3.6667 & 4.8214 & 0.7606 \\
\hline Octahedron & 8 & 3 & 8.2857 & 9 & 0.9206 \\
Hexagonal Lattice & 8 & 3 & 8.2857 & 9 & 0.9206 \\
\hline Icosahedron & 20 & 3 & 28.8421 & 21.0955 & 1.3672 \\
\hline Hexahedron & 6 & 4 & 5.2 & 5.0182 & 1.0362 \\
Square Lattice & 6 & 4 & 5.7714 & 5.8915 & 0.9796 \\
\hline Dodecahedron & 12 & 5 & 12.7273 & 11.1676 & 1.1397 \\
\hline \hline
\end{tabular}

TABLE 1: Analytic results for Platonic solids and planar analogues with periodic boundary conditions

\begin{tabular}{cc|ccc|cccc}
\hline \hline & & \multicolumn{4}{|c|}{ Markov Results } & \multicolumn{4}{|c}{ Monte Carlo Results } \\
$i$ & $N$ & $\langle n\rangle_{1}$ & $\langle n\rangle_{2}$ & $\Gamma$ & $\langle n\rangle_{1}$ & $\langle n\rangle_{2}$ & $\Gamma$ & $n_{\text {real }}$ \\
\hline 1 & 6 & 6.5 & 4.3860 & 1.4820 & 6.500 & 4.386 & 1.482 & $10^{7}$ \\
2 & 15 & 25.6857 & 16.0957 & 1.5958 & 25.686 & 16.096 & 1.596 & $10^{7}$ \\
3 & 42 & 118.0582 & 68.9220 & 1.7129 & 118.06 & 68.94 & 1.713 & $10^{6}$ \\
4 & 123 & - & - & - & 578.18 & 315.90 & 1.830 & $10^{5}$ \\
5 & 366 & - & - & - & 2886.1 & 1500.9 & 1.923 & $10^{3}$ \\
6 & 1095 & - & - & - & 14465.3 & 7282.9 & 1.986 & 10 \\
7 & 3282 & - & - & - & 72512.6 & 35724.1 & 2.029 & 1 \\
\hline \hline
\end{tabular}

TABLE 2: Walklength results for successive Sierpinski generation gaskets. 